\title{
An adaptive single-phase reclosing of line in the scheme with intermediate system
}

\author{
German Samorodov ${ }^{1}$, Tatyana Krasil'nikova ${ }^{1}$, and Saiyod Jononaev ${ }^{1, *}$ \\ ${ }^{1}$ Novosibirsk State Technical University, Automated Electrical Power Systems Department, 630073, Novosibirsk, Russian Federation
}

\begin{abstract}
Providing the reliability of adaptive single-phase reclosing (ASPR) in line equipped with any number of shunt reactors (SR), including the case of their absence, are considered and analyzed in this paper. The essence of the proposed work is that in order to provide high reliability of ASPR, is carry out short-term (for 0.1 seconds) one-sided switching on of the emergency phase from the side of the intermediate system, is measuring the current value of the current of this phase at the switched end and comparing it with the specified setpoint. If the current is higher than the specified setpoint, then a short or arc fault is detected in the phase and then it gives a command to disconnecting the line, otherwise it is not a short circuit in the phase and it is successfully will turned on to work on the other side of the line.
\end{abstract}

Keywords: adaptive single-phase reclosing, weak intermediate system, input resistance argument, controlled current in the emergency phase

The dead time of the SPR, was recommended on the basis of experimental data, is selected from the conditions for quenching the secondary arc and subsequent deionization of the arc gap. However, the true arc extinction time is often much less than that selected on the basis of the experimental curve. This circumstance reduces the effectiveness of SPR due to the overestimated effects of emergency automation. On the other hand, with stable single-phase faults (SPF) or a protracted secondary arc, unsuccessful SPR are not excluded. According to data of statistics, $15-30 \%$ of SPR may be unsuccessful, which increases the number of effects of short-circuit currents on power and switching equipment.

By the creation of powerful generating units and HV and EHV trunk lines, the problem of the successful elimination of the most probable single-phase faults has become more escalated. Really, an unsuccessful SPR worsens the operating conditions of circuit breakers that are at the limit of it interrupting capacity, on the other hand, the overestimated dead time of SPR on trunk lines requires an increase in the volume of emergency measures to provide the dynamic stability of power systems, which, in turn, leads to increased damage from the fault.

However, if you find a way to determine the time of extinction of the feed arc, then you can minimize the dead time of SPR for this accident associated with short circuit on the line. To achieve this goal, it is necessary to fix certain signs of the fact of self-destruction of the damage in the disconnected phase or the persistent nature of the damage.

Devices that determine the dead time of SPR in accordance with the true time of the existence of the feed arc are called adaptive devices for controlling the dead time of SPR, and SPR that satisfies this condition is called adaptive SPR. In the literature [1, 2, 3-11], it is proposed to control a number of operational parameters to determine the fact of the extinction of the feed arc and to decide on re-inclusion, such as:

- voltage module at the ends of the disconnected phase;

- voltage argument, measured in relation to a synchronously rotating vector;

- natural frequencies as part of the voltage of the disconnected phase;

- high-frequency components of the transition process during the burning of the feed arc;

- aperiodic current component in the neutral of the shunt reactor.

In the domestic literature [1, 2, 3-7], preference is given to monitoring the voltage of the disconnected phase to establish the fact of the extinction of the feed arc. If the voltage at the beginning of the emergency phase is selected as the controlled parameter, then it is necessary to compare the calculated situations shown in (Fig. 1).

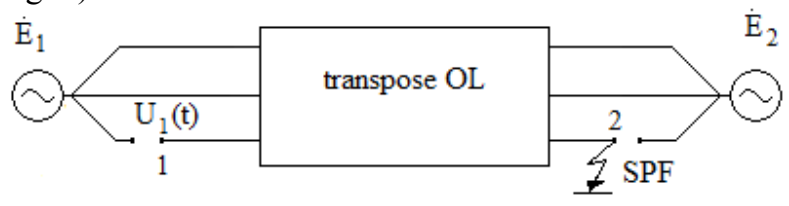

a)

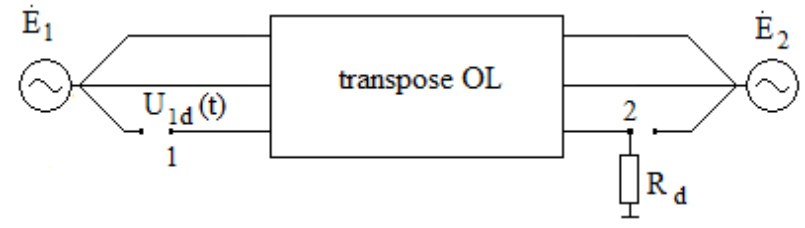

b)

\footnotetext{
* Corresponding author: jononaev.87@mail.ru
} 


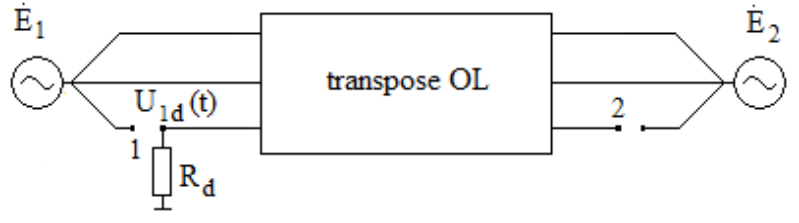

c)

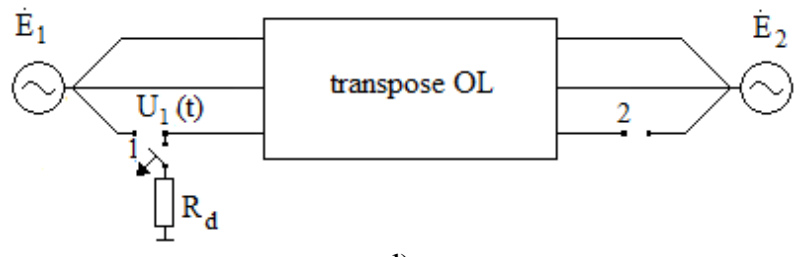

d)

Fig. 1. Comparable design situations with ASPR: a - stable SPF at the remote end of the overhead line $(\mathrm{OL}) ; \mathrm{b}-$ nonmissing secondary arc at the remote end of the overhead line; $\mathrm{c}$ - non-missing secondary arc at the controlled end of the overhead line; $\mathrm{d}$ - successful extinction of the secondary arc.

The developed ASPR reduce the likelihood of a phase being included at the final stage of ASPR on an sustained single-phase fault, but they do not completely exclude such a situation. So, for example, if one of the phases in the span to a tree is blocked, the arc will go out after the emergency phase is turned off, the gap strength will be restored, and ASPR will give a signal to turn on the phase. However, its inclusion will lead to repeated overlapping of the gap, since there is a hidden weak spot on the line that cannot be detected by using known methods of ASPR.

The method for implementing ASPR considered below, which is intended for use in intersystem connections that include a weak intermediate system (Fig. 2), is simple and allows to identify with a high degree of reliability all possible cases noted in Fig. 1. By an intermediate system we mean a weak system whose power is much less than the power of the connected systems.

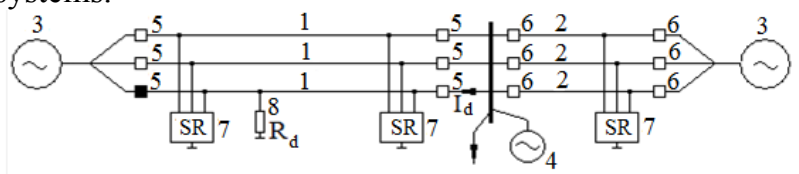

Fig. 2. ASPR of power lines in the presence of a weak intermediate system

The essence of the proposed solution is that after a two-sided disconnection of the damaged phase of the line for a selected dead time, a short-term (within 0.1 second) unilateral inclusion of the emergency phase from the side of the intermediate system 4 is carried out, the current value of the current of this phase at the switched on end is measured and compared with the specified setpoint.

The device contains phase lines 1 and 2 connected to systems 3 and 4 using switches 5 and 6 for any number of shunt reactors 7 on the lines. Any phase 1 or 2 may cause damage 8 .

The method is as follows. A two-way disconnected emergency phase can be in one of three states. When the damage resistance is $\mathrm{Rd} \approx 10 \mathrm{Ohm}$, an stable single- phase short circuit occurs, at $\mathrm{Rd} \approx 500 \mathrm{Ohm}$, an arc fault occurs and when $\mathrm{Rd} \rightarrow \infty$, there is no phase damage.

However, in the latter case, as noted above, there is no full guarantee that adaptive ASPR will be successful. Therefore, the two-sided disconnected emergency phase 1 is turned on one-sided from the side of the intermediate system 4 using the switch 5 , the current value of the current of this phase at the switched end is measured and compared with the specified setpoint. If the measured current is higher than the specified setpoint, then there is a short or arc fault in the phase, and a command to disconnect the line is given. Otherwise, there is no short circuit in the phase, and it successfully turns on at the other hand.

The specified current setting is selected from the condition of the detuning from the highest current, determined at the one-sided end of the phase in the absence of damage.

$$
I_{\mathrm{s}} \geq I_{\max } \cdot K_{r} \cdot K_{s}
$$

where $K_{r}=1,1$ - reliability coefficient, $K_{s}=1,25$ sensitivity coefficient.

Switching on from the side of the intermediate system 4 , the power of which, as a rule, is significantly less than the power of the adjacent systems 3 , is characterized by low short-circuit currents if the fault is maintained in phase and therefore does not pose a risk to the equipment.

The technical result is to provide a high degree of reliability of ASPR of power lines with any number of SR on the line. In addition, the method guarantees reliable identification of arc damage that cannot be detected on a two-sided emergency phase.

The diagram in Fig. 2 depicts the successful one-way switching on of the emergency phase of the line from the side of the intermediate substation at $\mathrm{Rd} \rightarrow \infty$. The mode parameters necessary for identifying the success of oneway switching are found according to an algorithm recorded in phase coordinates.

As a controlled value, you can use either the current from the side of the intermediate system, or the argument of the input impedance from the side of the intermediate system $\arg Z(\delta)$.

Figure 3 shows the dependence of the effective value of the monitored current of the emergency phase when it is turned on unilaterally from the side of the intermediate system from the angle.

The influence of the angle on the controlled current is insignificant, but the number of reactors connected to the line must be taken into account.

The number of reactors connected to the line has a smaller effect on the controlled parameter if the input impedance argument for the emergency phase from the side of the intermediate system is selected as the controlled parameter (Fig. 4). 


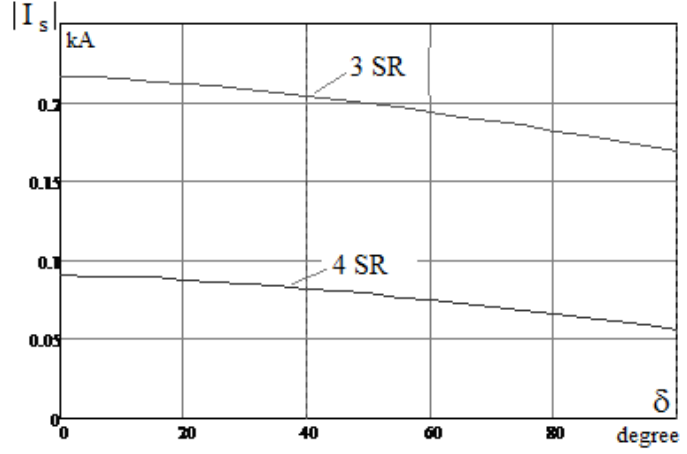

Fig. 3. Controlled current in the emergency phase from the side of the intermediate system with one-way switching on the overhead line

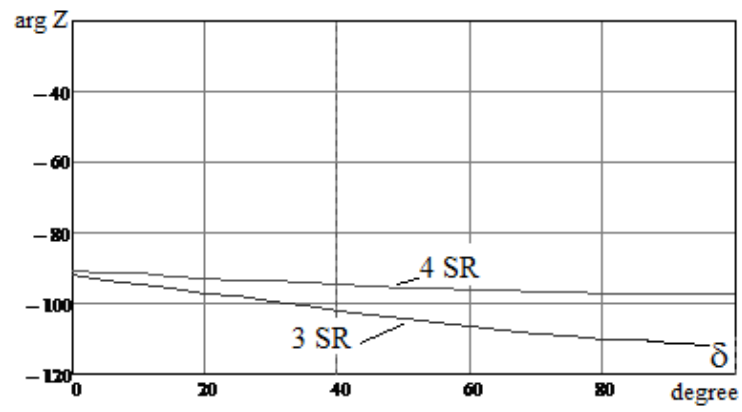

Fig. 4. Argument of input impedance for the emergency phase from the side of the intermediate system with one-way switching on the overhead line

The diagram in Fig. 2 depicts the one-way switching on of the emergency phase to a stable single-phase fault (at $\mathrm{Rd} \approx 10 \mathrm{Ohms}$ ) or to an arc fault (at $\mathrm{Rd} \approx 500 \mathrm{Om}$ ) of the line from the side of the intermediate substation.

Either the line current from system 4 or the input impedance argument from the side of the intermediate system is also used as a controlled quantity $\mathrm{Z}_{\mathrm{d}}\left(\delta, \mathrm{R}_{\mathrm{d}}\right)$.

Fig. 5 shows the dependences of the controlled current on the side of the intermediate system during an arc fault on the side of the open end of the overhead line

A significant difference in the currents at the beginning of the line in the modes of successful unilateral switching on of the emergency phase and its unilateral switching on the arc fault allows reliable identification of the outcome of the successful extinction of the secondary arc.

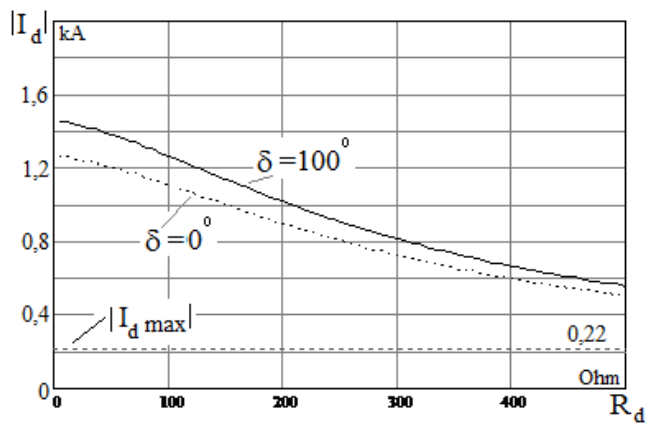

Fig. 5. Controlled current in the emergency phase from the side of the intermediate system depending on the arc resistance on a one-way switched on $500 \mathrm{kV}$ overhead line
The identification of a successful ASPR is more efficient by the argument of the input resistance relative to the buses of the controlled phase at the intermediate substation (Fig. 6).

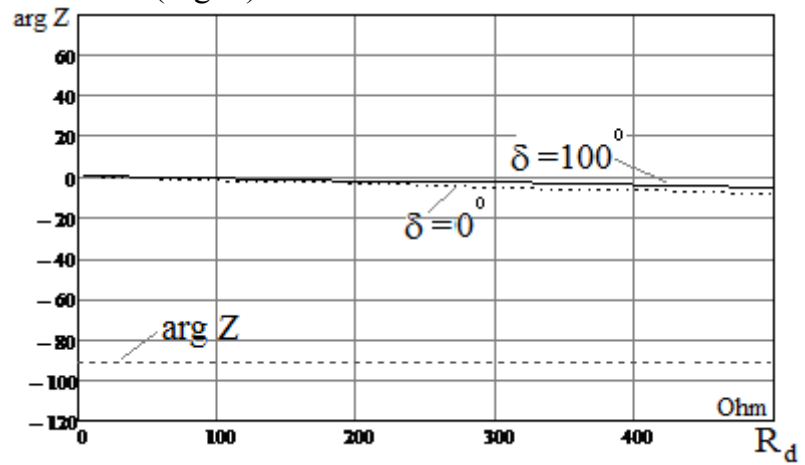

Fig. 6. Argument of the input impedance for the emergency phase from the side of the intermediate system depending on the arc resistance on a one-way switched on $500 \mathrm{kV}$ overhead

As the analysis showed, for EHV lines in the range of lengths of 100-500 km, depending on the number of SR on the line, the specified setpoint is no more than 0.25 $\mathrm{kA}$ for lines $500 \mathrm{kV}$ and $0.55 \mathrm{kA}$ for lines $750 \mathrm{kV}$, while possible the current range at the switched-on end of the emergency phase in the presence of a fault or arc is at least $0.8 \mathrm{kA}$ for $500 \mathrm{kV}$ lines and $1.2 \mathrm{kA}$ for $750 \mathrm{kV}$ lines, which is more than 3 times higher than the setting for $500 \mathrm{kV}$ lines and more than 2 times the setting for lines $750 \mathrm{kV}[8-11]$. Thus, there is a reliable guarantee of identification of damage.

Let us further consider how permissible a one-way switching on from the side of the intermediate system is under the condition that the single-phase fault current affects the adjacent system. It follows from the table that the current of a single-phase short circuit on the side of this system, when turned on unilaterally from the side of the intermediate system, depending on the location of the single-phase short circuit on the overhead line, is in the range of 3-4 kA. And this means that according to the table, these currents are approximately 3 times less than the single-phase short-circuit current in the initial circuit, i.e. not dangerous to the adjacent system.

Table. Normal mode and single-phase short-circuit currents

\begin{tabular}{|c|c|c|}
\hline $\begin{array}{c}\text { Normal } \\
\text { mode } \\
\text { current in } \\
\text { system 3, kA }\end{array}$ & $\begin{array}{c}\text { Single-phase } \\
\text { short current in } \\
\text { system } \mathbf{3} \text { for the } \\
\text { initial circuit, kA }\end{array}$ & $\begin{array}{c}\text { Single-phase short } \\
\text { current in system 3 } \\
\text { at unilateral inclusion } \\
\text { from the side of the } \\
\text { intermediate system, } \\
\text { kA }\end{array}$ \\
\hline 2 & 11 & $3-4$ \\
\hline
\end{tabular}




\section{Conclusion}

A new effective way of implementing ASPR based on a short-term one-side connection of the fault phase from the side of the intermediate system is proposed. Connecting from the side of an intermediate system, which has power significantly less than the power of adjacent systems, is characterized by low short-circuit currents if a single-phase short circuit is maintained by the phase and therefore does not pose a risk to equipment. The technical result is to provide a high of reliability of ASPR with any number of shunt reactors on the line. In addition, the method guarantees reliable identification of arc damage which cannot be detected on a two-sided disconnected faulted phase.

\section{References}

1. Aleksandrov G.N. The design of EHV transmission lines. Leningrad, Energoatomizdat Publ., 1993. 560 p.

2. D'iakov A.F. The electric networks of EHV and UHV UES of Russia. Theoretical and practical issues. Moscow, NTF "Energoprogress" Corporation "EEEK" Publ., in 3 vols., 2012. 696 p.

3. A. s. 1056341 USSR. MKI3 H02H 3/06. A device for monitoring the state of a phase of a power line with shunt reactors disconnected in the cycle of single-phase automatic reclosing / M.L. Levinstein, M.I. Khoroshev, A.F. Kalinichenko, Yu. I. Lyskov // Discovery. Inventions 1983 No. 46.

4. A. s. 1092642 USSR, MKM3 H02H 3/06. A method for controlling the disappearance of a short circuit on a disconnected power line and a device for its implementation / V. A. Ilyin, B. And Kovalev, M. And Khoroshev, Yu.I. Lyskov // Discoveries. Inventions 1984. No. 18.

5. A. s. 632023 USSR. MKI3 H02H 3/06. The method of single-phase automatic reclosing of the power line / V. N. Sobolev, V.F. Lachugin, Yu. I. Lyskov, V.G. Kagan // Discovery. Inventions 1978, No. 41.

6. A. s. 740108 USSR, MKM3 H02H 3/06 11/00. A method for monitoring the state of a power line phase disconnected in a single-phase automatic reclosing of a power line with shunt and compensation reactors / N. N. Belyakov, V. S. Pashkes, K. V. Hoscian // Discovery. Inventions 1981.№26.

7. S.P. Websper, A.T, Johns, R.K. Aggarwal. And R.W. Dunn «An investigation into breaker reclosure strategy for adaptive single pole autoreclosing», IEE Proc.- Gener. Transm. Distrib., vol.142, no.6, pp.601-607, Nov.1994.

8. S. Ahn, Ch.Kim, R.K. Aggarwal. and A.T, Johns, «An alternative approach to adaptive single pole autoreclosing in high voltage transmission systems based on variable dead time control», IEEE Trans. Power Delivery, vol.16, no.4, pp.676 - 686, Oct. 2001.

9. Krasil'nikova T.G., Dzhononaev S.G. Comparative analysis of ways to eliminate unstable single-phase short circuits in non-transposed lines of EHV and UHV// Electricity . 2017.no. 11, pp. 22-29.

10. Krasil'nikova T.G., Dzhononaev S.G. Effect of duration of pause SPAR on the throughput of the transmission in condition for dynamic stability.// Scientific Bulletin of Novosibirsk state technical University. 2017. no. 2 (67), pp.163-176.

11. Dzhononayev S.G. Elimination of unstable SinglePhase Short-Circuits in transposed lines in the SPAR cycle using automatic phase shunting // Scientific Bulletin of Novosibirsk state technical University. 2018. no. 3 (72), pp.157-174. 